\title{
Zero-Forcing Beamforming for Active and Passive Eavesdropper Mitigation in Visible Light Communication Systems
}

\author{
Sunghwan Cho, Student Member, IEEE, Gaojie Chen, Senior Member, IEEE, \\ and Justin P. Coon, Senior Member, IEEE
}

\begin{abstract}
This paper proposes zero-forcing (ZF) beamforming strategies that can simultaneously deal with active and passive eavesdroppers in visible light communication (VLC) systems. First, we propose a $\mathrm{ZF}$ beamforming scheme that steers a transmission beam to the null space of active eavesdroppers' (AEDs) channel, while simultaneously considering the SNRs for a legitimate user (UE) and passive eavesdroppers (PEDs) residing at unknown locations. To find an eigenmode related to the optimal beamforming vector, we adopt an inverse free preconditioned Krylov subspace projection method. For unfavorable VLC secrecy environments, the proposed ZF beamformer appears to be incapable of effectively coping with the PEDs due to the strict condition that the data transmission must be in the null space of the AEDs' channel matrix. Hence, an alternative beamforming scheme is proposed by relaxing the constraint on the SNRs of the AEDs. The related optimization problem is formulated to reduce the secrecy outages caused by PEDs, while simultaneously satisfying the target constraints on the SNRs of the UE and the AEDs. To simplify the mathematical complexity of the approach, Lloyd's algorithm is employed to sample the SNR field, which in turn discretizes the problem, thus making it tractable for practical implementation. The numerical results show that both the exact and relaxed $\mathrm{ZF}$ beamforming methods achieve superior performance in the sense of secrecy outage relative to a benchmark $Z F$ scheme. Moreover, the proposed relaxed $\mathrm{ZF}$ beamforming method is shown to cope with PEDs better than the exact $\mathrm{ZF}$ beamforming approach for unfavorable VLC environments.
\end{abstract}

Index Terms-Physical layer security, visible light communication, beamforming, active eavesdroppers, passive eavesdroppers.

\section{INTRODUCTION}

Visible light communication (VLC) has gained considerable popularity as a feasible solution to resolve the shortfall of radio frequency (RF) spectrum by utilizing visible light spectrum for wireless communications. VLC has many advantages in terms of unlicensed wide bandwidth, low implementation cost, and high area spectral efficiency. Moreover, thanks to the inherent characteristics of visible light, namely that it cannot penetrate opaque walls, VLC can provide high security [1].

Nevertheless, VLC still retains the possibility of being eavesdropped in large open (public) spaces, such as open offices, shopping malls, libraries, and hospitals. Therefore,

S. Cho and J. P. Coon are with the Department of Engineering Science, University of Oxford, Oxford, OX1 3PJ, U.K. (e-mail: \{sunghwan.cho, justin.coon\}@eng.ox.ac.uk).

G. Chen is with the Department of Engineering, University of Leicester, Leicester, LE1 7RH, U.K. (e-mail: gaojie.chen@leicester.ac.uk). various physical layer security (PLS) techniques have been studied with a view to securing VLC systems by tailoring RF PLS techniques to VLC environments [2]. PLS is a set of techniques that secure wireless transmissions by utilizing the channel randomness between the transmitter and the receiver [3].

Many existing PLS methods for VLC systems - such as beamforming, jamming, and light-emitting diode (LED) selection, relay-aided transmission [4]-[10] - are based on the assumption that either active or passive eavesdroppers (EDs) are present, but not both simultaneously. The difference between active and passive EDs in VLC systems lies with the availability of the channel state information (CSI) of the EDs at the transmitter sites. In reality, it may be the case that EDs try to escape the vigilance of a legitimate user (UE) or a network manager; thus, it would be challenging to obtain information about the types, locations, and numbers of the EDs. The common assumption on EDs is that only active EDs (AEDs) are present. In general, the objective of PLS schemes based on this assumption is to suppress the signal received at the AEDs' sites by steering a transmission beam to the null space of the AEDs' channels [5]-[7]. However, if unknown passive EDs (PEDs) are concurrently present, these PLS schemes may yield opportunities for eavesdropping at the PEDs' sites.

In order to clearly explain the vulnerability of existing PLS schemes, an exemplary result corresponding to a zero-forcing (ZF) scheme [5] is provided in Fig. 1. The ZF beamformer eliminates information reception by an unintended user (i.e., an AED) by designing the precoding matrix such that the transmitted signal lies in the null space of the AED's channel. The figure shows the signal-to-noise ratios (SNRs) according to measurement locations when the ZF strategy is adopted. One UE and one AED are assumed to be present; however, any PEDs that may reside in the neighborhood of the transmission are ignored. The UE and the AED are marked with blue and green dots, respectively, in the figure.

In Fig. 1, we note that even when the AED is located close to the UE, the ZF beamformer effectively minimizes the information reception at the AED site. From this result, the ZF strategy appears to secure the transmission. However, from the perspective of any passive devices that may exist in the region, it is possible to achieve a higher SNR than the UE. Note that if any PED is located in the black contoured area in Fig. 1, it can achieve a higher SNR than the UE, 


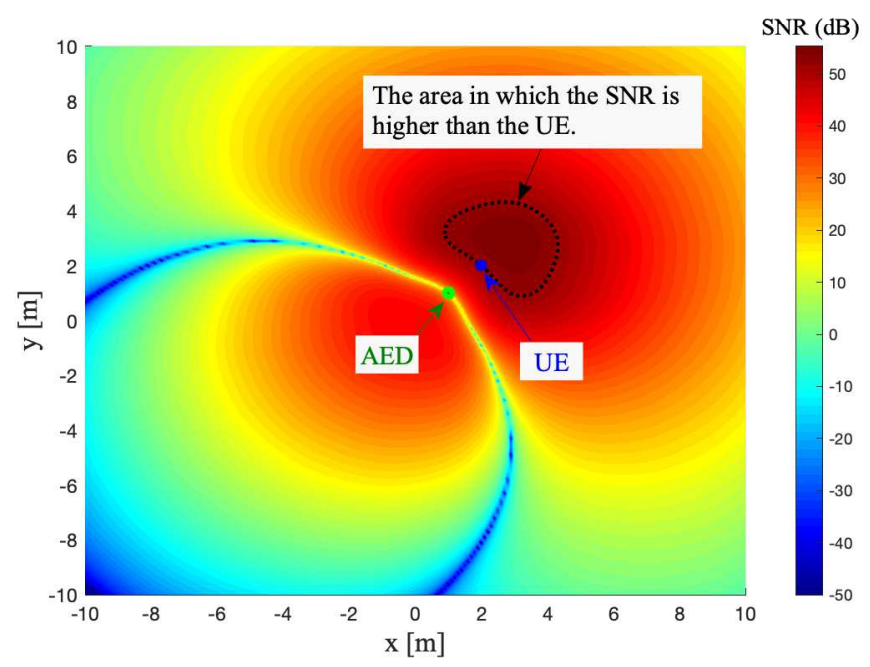

Fig. 1. The SNR according to the receiver locations when ZF precoding is utilized. The UE and the AED are located at $(2,2)$ and $(1,1)$, respectively.

which results in a secrecy outage. Compared to beamforming and LED selection schemes devised for transmission in the presence of PEDs, for which the UE is capable of retaining the highest SNR [8], the ZF precoder designed for the nearby AED expands the contour area where the SNR is higher than that of the UE. This more spacious area would yield more opportunities for eavesdropping to the PEDs, which results in a higher secrecy outage probability (SOP). This vulnerability originates from the fact that the ZF design purely aims at forcing the AED's SNR to zero without consideration for SNRs of other unknown receivers in the area. In contrast, since the previous PLS schemes devised for PEDs [8], [9] have not considered AEDs, which can be regarded as more apparent security threats than PEDs, the PLS schemes need to be further improved to cope with any types of EDs.

This paper is the first work to propose a novel ZF beamforming strategy that can deal with both active and passive EDs. In order to take the unknown (passive) ED locations into account for the beamforming, we utilize tools from stochastic geometry as in our previous works [8], [9]. This paper begins with proposing a ZF beamforming strategy that minimizes the SNRs of the AEDs while maximizing the SNR of the UE and constraining the average SNR of the PEDs. An optimization problem is formulated to find a beamforming vector that lies in the null space of the AEDs, satisfying the requirements and constraints for the UE and the PEDs. The proposed beamforming strategy directs the transmission along a particular eigenmode related to the null space of the active ED channels and the intensity of the passive ED point process. An inverse free preconditioned Krylov subspace projection method [11] is adopted to find the eigenmode. The numerical results show that the proposed ZF beamforming strategy can effectively secure the VLC transmission against active and passive EDs.

On the other hand, in some cases, especially when the number of LED transmitters is small and the location of the UE is
TABLE I. Notation and symbols used in the paper.

\begin{tabular}{|c|l|}
\hline Symbol & Definition/Explanation \\
\hline$L$ & the length of a room \\
$H$ & the width of a room \\
$N$ & the height from the ceiling to the work plane \\
$N_{A}$ & number of transmitters \\
$\alpha$ & moduer of active eavesdroppers \\
$I_{D C}$ & fixed bias current \\
$\eta$ & current-to-light conversion efficiency \\
$\phi_{1 / 2}$ & half illuminance angle \\
$A_{\mathrm{PD}, k}$ & physical area of a photodiode \\
$\phi_{i, k}$ & angle of irradiance \\
$\psi_{i, k}$ & angle of incidence \\
$\kappa_{k}$ & refractive index of an optical concentrator \\
$\Psi_{k}$ & received field of view of a photodiode \\
$R_{k}$ & photodetector's responsivity \\
$T_{k}$ & transimpedance amplifier gain \\
$\mathbb{R}$ & set of real numbers \\
$\mathbb{R}_{+}$ & set of non-negative real numbers \\
$\mathbb{1}$ & all-ones column vector \\
$\mathbb{O}$ & all-zeros column vector \\
$\mathbb{E}[\cdot]$ & expectation operator \\
{$[\cdot]^{T}$} & transpose operator \\
$\mathbb{P}(\cdot)$ & probability operator \\
&
\end{tabular}

not favorable from the secrecy perspective, the strict condition that the data transmission must be in the null space of the AEDs' channel matrix makes the proposed ZF beamforming strategy unable to cope with the PEDs effectively. Thus, an alternative solution is proposed to relax the constraint on the SNRs of the AEDs and more rigorously suppress the SNRs of PEDs. Thus, we call this the relaxed $Z F$ beamforming scheme. Accordingly, we formulate an optimization problem with the objective to minimize the SOP by reducing the area in which the SNR is higher than that of the UE, thus reducing the likelihood that PEDs will achieve a higher SNR than the UE. To simplify the calculation of the area, we adopt an approach of considering the SNRs measured at discrete points obtained by the Lloyd algorithm [12]. The optimization also has constraints that regulate both the SNRs of the UE and the AEDs.

The rest of this paper is organized as follows ${ }^{1}$. Section II begins with the system model describing the modulation and beamforming schemes in VLC and providing various performance measures. In Section III, the ZF beamforming scheme maximizing the SNR of the UE and constraining the average SNR of the PEDs is investigated. In Section IV, the relaxed ZF beamforming approach is proposed. Section V gives numerical results that support our analysis. Section VI concludes the paper.

\footnotetext{
${ }^{1}$ The notation and symbols used in the paper are listed in Table I.
} 


\section{SYSTEM MODEL}

\section{A. Data Transmission}

We consider the downlink of a VLC system as shown in Fig. 2, where $W, L$, and $H$ denote the width, the length, and the height of the ceiling relative to the work plane, respectively. $N$ LED transmitters are attached to the ceiling of the room. Each transmitter - i.e., an LED fixture consisting of multiple individual LEDs - is assumed to be capable of communicating independently of other transmitters. We assume that one UE and $N_{\mathrm{A}}$ AEDs are present in the work plane of the room. Multiple PEDs are assumed to be randomly distributed according to a homogeneous Poisson point process (PPP) $\Phi_{P E}$ with density $\lambda_{P E}$. Note that the uniform distribution of the PEDs means that their locations and CSI are completely random and unknown, which is the worst case from the secrecy viewpoint [13]. On the other hand, the CSI of the UE and the AEDs are known to the LED transmitters. The UE, the AEDs, and the PEDs are denoted by $U, A E_{p}$ for $p \in\left\{1,2, \ldots, N_{\mathrm{A}}\right\}$, and $P E_{q}$, respectively. Also, we assume that EDs act independently of one another, i.e., there is no collusion.

A direct-current (DC) biased pulse-amplitude modulation VLC scheme is considered [4]. The data signal $s_{i}(t) \in \mathbb{R}$ for the $i$ th transmitter in time slot $t$ is superimposed on a fixed bias current $I_{D C} \in \mathbb{R}_{+}$. The current $I_{D C}$ is used for the purpose of illumination. Thus, the $i$ th transmitter's modulated signal $x_{i}(t)$ corresponding to $s_{i}(t)$ is a zero-mean current signal that can be expressed as $x_{i}(t)=\alpha I_{D C} s_{i}(t)$, where $\alpha \in[0,1]$ is the modulation index. To maintain linear current-to-light conversion, the amplitude of $x_{i}(t)$ is constrained such that $\left|x_{i}(t)\right| \leq \alpha I_{D C}$. Also, $\mathbb{E}\left[x_{i}(t)\right]=0$ is assumed; thus the data signal does not affect illumination.

According to [14], the channel gain $h_{i, k} \in \mathbb{R}_{+}$from the $i$ th transmitter to receiver $k \in\left\{U, A E_{p}, P E_{q}\right\}$ corresponding to an LED with a generalized Lambertian emission pattern in a VLC system is given by $h_{i, k}=\eta G_{i, k} R_{k} T_{k}$, where $\eta$ (W/A) is the current-to-light conversion efficiency, $R_{k}$ $(\mathrm{mA} / \mathrm{mW})$ is the photodetector's responsivity, and $T_{k}(\mathrm{~V} / \mathrm{A})$ is the transimpedance amplifier gain. Notice that this channel model considers only LoS propagation and ignores reflections from walls and windows. Such a model is justified for most indoor scenarios in which LEDs are attached to the ceiling and facing down, making reflections significantly weaker than LoS components [14]. Moreover, note that if an advanced modulation scheme that combats inter-symbol interference, e.g., optical orthogonal frequency division multiplexing, is employed, multipath reflections can be neglected [15]. The Line-of-Sight (LoS) path gain $G_{i, k}$ is given by

$$
\begin{aligned}
& G_{i, k}= \\
& \left\{\begin{array}{cll}
\frac{(m+1) A_{\mathrm{PD}, k}}{2 \pi l_{i, k}^{2}} \frac{\kappa_{k}^{2} \cos ^{m}\left(\phi_{i, k}\right)}{\sin ^{2}\left(\Psi_{c}\right)} \cos \left(\psi_{i, k}\right) & \text { for } & \left|\psi_{i, k}\right| \leq \Psi_{c}, \\
0 & \text { for } & \left|\psi_{i, k}\right|>\Psi_{c}
\end{array}\right.
\end{aligned}
$$

where $m=-\ln (2) / \ln \left(\cos \left(\phi_{1 / 2}\right)\right)$ is the order of Lambertian emission with half illuminance at $\phi_{1 / 2}$, and $A_{\mathrm{PD}, k}\left(\mathrm{~m}^{2}\right)$ is

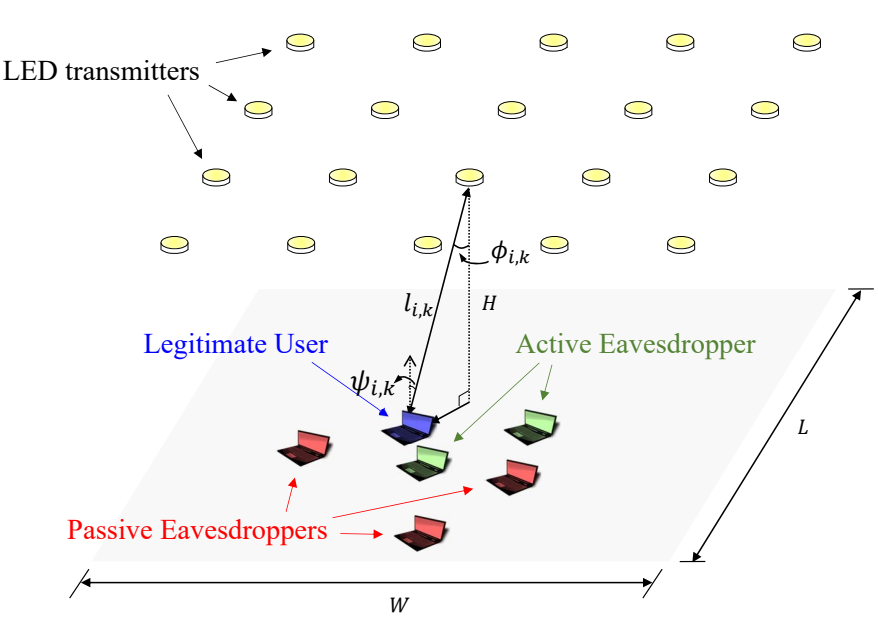

Fig. 2. Rectangular room configuration for VLC systems.

the physical area of the photodiode (PD) at the receiver. As shown in Fig. $2, l_{i, k}(\mathrm{~m})$ denotes the distance between the $i$ th transmitter and receiver $k . \phi_{i, k}$ is the angle of irradiance, and $\psi_{i, k}$ is the angle of incidence. $\kappa_{k}$ is the refractive index of the optical concentrator, and $\Psi_{c}$ denotes the received field of view of the PD. Moreover, by assuming that a receiver's PD faces up normal to the work plane, i.e., assuming $\phi_{i, k}=\psi_{i, k}$, we can rewrite the channel gain as $h_{i, k}=K l_{i, k}^{-(m+3)}$, where $K=\left(\eta(m+1) A_{\mathrm{PD}, k} H^{m+1} \kappa_{k}^{2} R_{k} T_{k}\right) /\left(2 \pi \sin ^{2}\left(\Psi_{c}\right)\right)$.

As in [4], [8], we define a multiple-input single-output (MISO) beamforming vector $\mathbf{w}=\left[\omega_{1}, \omega_{2}, \ldots, \omega_{N}\right]^{T}$, where $\omega_{i} \in \mathbb{R}$ for $i \in\{1,2, \ldots, N\}$ is a weight for the $i$ th transmitter and $\left|\omega_{i}\right| \leq 1$. The $i$ th LED transmitter multiplies the modulated data signal $x_{i}(t)$ by $\omega_{i}$ before transmitting. In the considered MISO VLC system, the data signal $s_{i}(t)$ for the $i$ th transmitter is assumed to be identically $s(t)$ for all transmitters as in [4]. Therefore, the signal at the receiver $k$ in the MISO VLC system can be described as

$$
y_{k}(t)=\alpha I_{D C} \mathbf{h}_{k}^{T} \mathbf{w} s(t)+n_{k}(t)
$$

where $\mathbf{h}_{k}=\left[h_{1, k}, h_{2, k}, \ldots, h_{N, k}\right]^{T} \in \mathbb{R}^{N}$ denotes the channel gain vector. Also, $n_{k}$ signifies a zero-mean additive white Gaussian noise (AWGN) random variable with variance $\sigma^{2}$ at receiver $k$. We assume that all the receivers retain an identical AWGN power variance $\sigma^{2}$. For notational convenience, the time index $t$ is ignored for the remainder of the paper.

\section{B. Performance Measures}

For Gaussian VLC MISO channels with an amplitude constraint, the $\mathrm{SNR}^{2}$ at receiver $k$ with beamforming can be written as

$$
\gamma_{k}=\frac{\alpha^{2} I_{D C}^{2} \mathbf{w}^{T} \mathbf{h}_{k} \mathbf{h}_{k}^{T} \mathbf{w}}{\sigma^{2}}=\varphi \mathbf{w}^{T} \mathbf{H}_{k} \mathbf{w}
$$

\footnotetext{
${ }^{2}$ In fact, this expression is the peak SNR of the link; we use the term $S N R$ to refer to the peak SNR throughout the paper for brevity.
} 
where $\mathbf{H}_{k}=\mathbf{h}_{k} \mathbf{h}_{k}^{T}$ and $\varphi=\alpha^{2} I_{D C}^{2} / \sigma^{2}$. The average SNR of a PED can be expressed as

$$
\begin{aligned}
\bar{\gamma}_{P E} & =\mathbb{E}\left[\varphi \mathbf{w}^{T} \mathbf{h}_{P E_{q}} \mathbf{h}_{P E_{q}}^{T} \mathbf{w}\right] \\
& =\varphi \mathbf{w}^{T} \mathbb{E}\left[\mathbf{h}_{P E_{q}} \mathbf{h}_{P E_{q}}^{T}\right] \mathbf{w}=\varphi \mathbf{w}^{T} \overline{\mathbf{H}}_{P E} \mathbf{w}
\end{aligned}
$$

where $\overline{\mathbf{H}}_{P E}=\mathbb{E}\left[\mathbf{h}_{P E_{q}} \mathbf{h}_{P E_{q}}^{T}\right]$ and its element in the $i$ th row and $j$ th column is given by

$$
\begin{aligned}
\bar{H}_{P E_{i, j}} & =\mathbb{E}\left[h_{i, P E_{q}} h_{j, P E_{q}}\right] \\
& =\mathbb{E}\left[K^{2} l_{i, P E_{q}}^{-(m+3)} l_{j, P E_{q}}^{-(m+3)}\right] \\
& =\frac{1}{L W} \int_{\frac{-L}{2}}^{\frac{L}{2}} \int_{\frac{-W}{2}}^{\frac{W}{2}} \frac{K^{2}}{l_{i}^{m+3}(\mathbf{x}) l_{j}^{m+3}(\mathbf{x})} \mathrm{d} \mathbf{x}
\end{aligned}
$$

where $l_{i}(\mathbf{x})=\sqrt{\left|\mathbf{x}-\mathbf{x}_{i}^{\mathrm{LED}}\right|^{2}+H^{2}}$ denotes the distance between the $i$ th LED transmitter and the point $\mathbf{x} \in \mathbb{R}^{2}$ in the work plane of the room (its origin is the center of the room), where $\mathbf{x}_{i}^{\mathrm{LED}} \in \mathbb{R}^{2}$ for $i \in\{1,2, \ldots, N\}$ is the location of the $i$ th LED transmitter translated to the work plane.

The SOP can be described by the equation

$$
P_{S O} \stackrel{(a)}{=} \mathbb{P}\left(\gamma_{U}<\max _{\mathbf{x} \in \Phi_{P E}} \gamma(\mathbf{x}, \mathbf{w})\right) \stackrel{(b)}{=} 1-\exp \left(-\iint_{\mathcal{A}} \lambda_{P E} \mathrm{~d} \mathbf{x}\right)
$$

where $\gamma(\mathbf{x}, \mathbf{w})$ denotes the SNR measured at the point $\mathbf{x}$, which can be expressed as

$$
\gamma(\mathbf{x}, \mathbf{w})=\varphi \mathbf{w}^{T} \mathbf{h}(\mathbf{x}) \mathbf{h}(\mathbf{x})^{T} \mathbf{w}
$$

where $\mathbf{h}(\mathbf{x})$ denotes the channel gain vector at the point $\mathbf{x}$. For (a), we assume that secrecy outage only occurs when the maximum SNR observed by a PED is greater than the SNR of the UE, which is a justifiable assumption since the SNRs of the AEDs are effectively minimized by using the proposed beamforming design. For (b), we apply the void probability of a PPP [17], where $\mathcal{A}=\left\{\mathbf{x} \mid \gamma(\mathbf{x}, \mathbf{w})>\gamma_{U}\right\}$. Note that $\mathcal{A}$ denotes the black contour area in Fig. 1.

\section{ZERO-FORCING BEAMFORMING}

To design the beamforming scheme that simultaneously copes with the AEDs and the PEDs, we exploit the traditional ZF scheme for VLC systems [5]. The traditional ZF beamformer for a single AED $A E_{1}$, where the PEDs are ignored, can be obtained by projecting the UE's channel onto the null space of the AED [18]. The corresponding ZF beamforming vector can be expressed as

$$
\mathbf{w}=g \Psi_{A E_{1}} \Psi_{A E_{1}}^{T} \mathbf{h}_{U}
$$

Here, $\Psi_{A E_{1}} \in \mathbb{R}^{N \times(N-1)}$ is a matrix whose $N-1$ columns constitute a basis for the null space of the AED's channel vector $\mathbf{h}_{A E_{1}}$, and $g$ is a constant such that $|\mathbf{w}| \leq \mathbb{1}$. Note that by constraining the beamforming vector to lie in the null space of the AED channel, the SNR of the AED $\gamma_{A E_{1}}$ can be minimized. Based on this idea, in this section, we find a new ZF beamformer that lies in the null space of the AEDs' channels in order to minimize the SNRs of the AEDs while simultaneously considering the SNRs of the UE and the PEDs.

\section{A. Maximizing the Legitimate User's SNR}

First, we formulate the optimization problem for the ZF beamforming vector $\mathbf{w}$ that maximizes the SNR of the UE while constraining the average SNR of the PEDs. Without knowledge of PED locations, considering the average SNR of the PEDs is a natural approach [8], [9]. In addition, it is worth noting that the secrecy outages caused by PEDs can also be reduced by maximizing the SNR of the UE given a constraint on the average SNR of the PEDs.

In a similar manner to (8), we constrain the beamforming vector $\mathbf{w}$ to lie in the null space of the AEDs' channel matrix $\left[\begin{array}{llll}\mathbf{h}_{A E_{1}} & \mathbf{h}_{A E_{2}} & \ldots & \mathbf{h}_{A E_{N_{A}}}\end{array}\right]^{T}$, which can be expressed as

$$
\mathbf{w}=\Psi_{A E} \Psi_{A E}^{T} \mathbf{a}
$$

where $\Psi_{A E} \in \mathbb{R}^{N \times\left(N-N_{A}\right)}$ is a matrix whose $N-N_{A}$ columns constitute a basis for the null space of the AEDs' channel matrix, and $\mathbf{a} \in \mathbb{R}^{N}$ is an arbitrary real vector. This condition ensures that the beamforming vector $\mathbf{w}$ derived from the projection makes the SNRs of the AEDs minimized. Therefore, letting $\Theta=\Psi_{A E} \Psi_{A E}^{T}$, i.e., $\mathbf{w}=\Theta \mathbf{a}$, the optimization problem involving the vector a can be written as

$$
\begin{aligned}
& \arg \max _{\mathbf{a}} \varphi \mathbf{a}^{T} \mathbf{H}_{U}^{\Theta} \mathbf{a} \\
& \text { s.t. }\left\{\begin{array}{l}
\varphi \mathbf{a}^{T} \overline{\mathbf{H}}_{P E}^{\Theta} \mathbf{a} \leq \bar{\rho}_{P E} \\
|\Theta \mathbf{a}| \leq \mathbb{1}
\end{array}\right.
\end{aligned}
$$

where $\mathbf{H}_{U}^{\Theta}=\Theta^{T} \mathbf{H}_{U} \Theta$ and $\overline{\mathbf{H}}_{P E}^{\Theta}=\Theta^{T} \overline{\mathbf{H}}_{P E} \Theta$, and $\bar{\rho}_{P E}$ denotes the target constraint on the average SNR of a $\mathrm{PED}^{3}$. The Lagrangian for this problem is

$$
\begin{array}{r}
\mathcal{L}=\varphi \mathbf{a}^{T} \mathbf{H}_{U}^{\Theta} \mathbf{a}-\lambda\left(\varphi \mathbf{a}^{T} \overline{\mathbf{H}}_{P E}^{\Theta} \mathbf{a}-\bar{\rho}_{P E}\right) \\
+\mu_{-}^{T}(\Theta \mathbf{a}+\mathbb{1})-\mu_{+}^{T}(\Theta \mathbf{a}-\mathbb{1})
\end{array}
$$

where $\lambda \in \mathbb{R}$ and $\mu_{-}, \mu_{+} \in \mathbb{R}^{N}$ are the Lagrange multipliers. For $\mathcal{L}$ to yield a non-trivial, analytic maximum with respect to a, we assume the second constraint of (10b) is slack, i.e., any element of $\Theta \mathbf{a}$ is strictly in between -1 and 1 . Then, we have

$$
\mu_{-}=\mu_{+}=\mathbb{O}
$$

according to the local sensitivity analysis in [19]. The satisfaction of the condition stated in (12) depends upon $\bar{\rho}_{P E}$. If $\bar{\rho}_{P E}$ is small, no element of $\Theta \mathbf{a}$ will reach \pm 1 , i.e., the second constraint is inactive.

It is worth noting that $\bar{\rho}_{P E}$ is a system parameter to be appropriately chosen according to given VLC environments, such as a particular distribution of LED transmitters, the location of the UE, the LED transmit power, etc. For example, if $\bar{\rho}_{P E}$ is set too low, the LED transmitters should reduce the transmit power to satisfy the $\bar{\rho}_{P E}$ constraint, but the received signal power at the UE site would also be reduced in a commensurate manner. On the other hand, if the UE is located very close to one of the LED transmitters, the closest

\footnotetext{
${ }^{3}$ For PEDs distributed by homogeneous PPP, considering the average SNR of a single PED $\bar{\gamma}_{P E}$ is identical to constraining the sum of the average SNRs of multiple PEDs since they obey the relation $\mathbb{E}\left[\sum \gamma_{P E_{q}}\right]=\lambda_{P E} L W \bar{\gamma}_{P E}$ due to Campbell's theorem [16].
} 
LED transmitter to the UE can increase its transmit power to increase the SNR of the UE while simultaneously satisfying the $\bar{\rho}_{P E}$ constraint. PLS theories suggest choosing the value of $\bar{\rho}_{P E}$ in a way that makes the SNR difference between the UE and the PEDs as large as possible [3]. To obtain the large SNR gap, it is highly likely that the UE receives the information signal mainly from the nearest LED transmitter; in contrast, the other far-off transmitters transmit with less power or be silent. This is because, due to the significant dependence of the VLC channel gain on the distance, the transmissions from the LEDs far from the UE would not significantly contribute to the SNR of the UE, while it would more considerably increase the SNRs of PEDs that might be present in the entire room. Thus, in practice, it would be essential to set $\bar{\rho}_{P E}$ such that only the nearest LED transmits the information with a beamforming weight close to \pm 1 , while the other LEDs' beamforming weights are much less or large than \pm 1 , respectively. In this sense, when engineering the secured VLC system, the condition (12) is likely to be satisfied.

Then, with assuming that (12) is satisfied, setting the partial derivative of $\mathcal{L}$ (with respect to a) equal to zero leads to

$$
\mathbf{H}_{U}^{\Theta} \mathbf{a}=\lambda \overline{\mathbf{H}}_{P E}^{\Theta} \mathbf{a} .
$$

Here, $\overline{\mathbf{H}}_{P E}^{\Theta}$ is singular with rank less than or equal to $N-N_{A}$. Hence, we employ the inverse free preconditioned Krylov subspace projection method [11] to obtain the maximum eigenvalue $\lambda_{\max }$ and corresponding eigenvector $\mathbf{a}_{\max }$. In this method, an eigenvector estimate $\mathbf{a}_{k}$, where $k$ denotes the iteration number, iteratively improves through the RayleighRitz projection onto the Krylov subspace

$$
\begin{aligned}
& K_{m}\left(\mathbf{H}_{U}^{\Theta}-\rho_{k} \overline{\mathbf{H}}_{P E}^{\Theta}, \mathbf{a}_{k}\right) \\
& =\operatorname{span}\left(\mathbf{a}_{k},\left(\mathbf{H}_{U}^{\Theta}-\rho_{k} \overline{\mathbf{H}}_{P E}^{\Theta}\right) \mathbf{a}_{k}, \ldots,\left(\mathbf{H}_{U}^{\Theta}-\rho_{k} \overline{\mathbf{H}}_{P E}^{\Theta}\right)^{m} \mathbf{a}_{k}\right)
\end{aligned}
$$

where $\rho_{k}=\mathbf{a}_{k}^{T} \mathbf{H}_{U}^{\Theta} \mathbf{a}_{k} / \mathbf{a}_{k}^{T} \overline{\mathbf{H}}_{P E}^{\Theta} \mathbf{a}_{k}$ denotes the Rayleigh quotient, and $m$ is a parameter to be chosen. $\operatorname{span}\left(\mathbf{v}_{1}, \mathbf{v}_{2}, \ldots, \mathbf{v}_{N}\right)$ denotes the set of all linear combinations of a collection of vectors $\mathbf{v}_{1}, \mathbf{v}_{2}, \ldots, \mathbf{v}_{N}$. In this paper, we use the MATLAB program eigifp implemented in [20], which can carry out the whole procedure mentioned above by taking the two inputs, $\mathbf{H}_{U}^{\Theta}$ and $\overline{\mathbf{H}}_{P E}^{\Theta}$, and returning the maximum eigenvalue and corresponding eigenvector.

The solution of (13) satisfies

$$
\gamma_{U}=\varphi \mathbf{a}^{T} \mathbf{H}_{U}^{\Theta} \mathbf{a}=\varphi \lambda \mathbf{a}^{T} \overline{\mathbf{H}}_{P E}^{\Theta} \mathbf{a} \leq \lambda_{\max } \bar{\rho}_{P E} .
$$

Here, it is noted that the maximum SNR of the UE is proportional to $\lambda_{\max }$. Therefore, the optimal vector $\mathbf{a}^{*}$ can be obtained by scaling the eigenvector $\mathbf{a}_{\max }$ corresponding to $\lambda_{\max }$ such that $\varphi \mathbf{a}^{* T} \overline{\mathbf{H}}_{P E}^{\Theta} \mathbf{a}^{*}=\bar{\rho}_{P E}$. The optimal beamforming vector $\mathbf{w}^{*}$ is given by $\mathbf{w}^{*}=\Theta \mathbf{a}^{*}$. Lastly, the assumption that (12) is satisfied must be verified to confirm the feasibility of $\mathbf{w}^{*}$. If any element of $\mathbf{w}^{*}$ is strictly in between -1 and $1,(12)$ is satisfied; thus the acquired solution $\mathbf{w}^{*}$ is confirmed to be the optimal solution.

On the other hand, if this condition (12) is not satisfied,

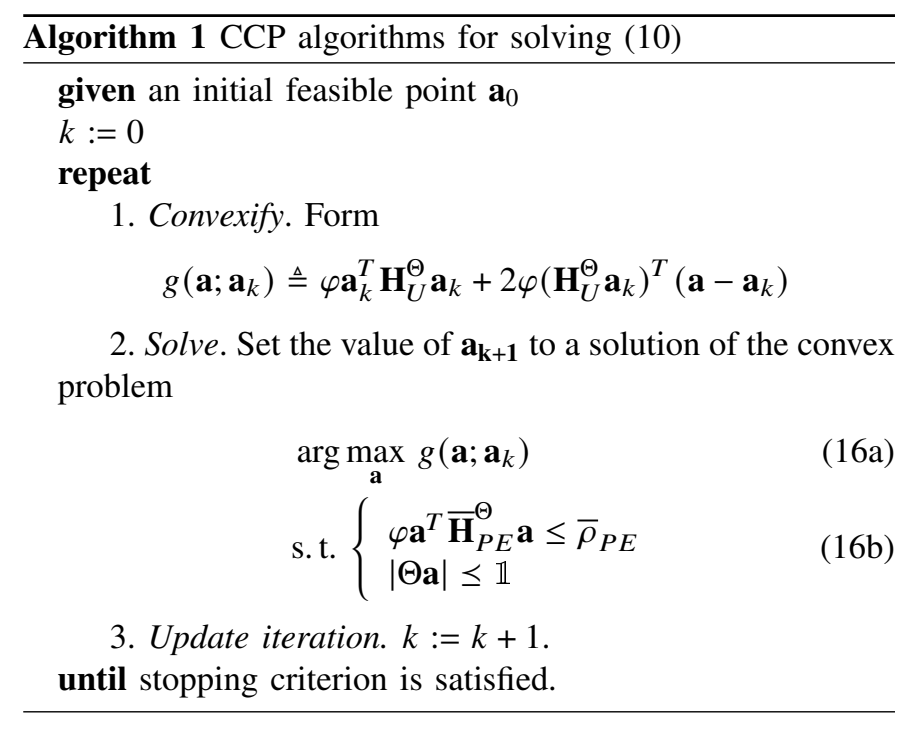

the solution $\mathbf{a}^{*}$ can be numerically found with the convexconcave procedure (CCP) [21], which is a powerful heuristic method to find local solutions. Given an initial feasible point $\mathbf{a}_{0}$, we transform the objective function (10a) into an affine function (i.e., convex) by applying the first-order Taylor series approximation and solve the convex optimization problem by using a standard optimization programming method like the sequential quadratic programming (SQP) algorithm [22]. Then, we set the solution to $\mathbf{a}_{1}$ and repeat the same procedure until the improvement in the objective value is less than some threshold $\epsilon$. The detailed iterative algorithm is given in Algorithm 1.

\section{B. Minimizing the Average SNR of Passive Eavesdroppers}

We now investigate the problem of finding a ZF beamforming vector that minimizes the average SNR of the PEDs while satisfying the required SNR of the UE. Similar to the previous subsection, we can formulate the optimization problem in terms of a:

$$
\begin{aligned}
& \arg \min _{\mathbf{a}} \varphi \mathbf{a}^{T} \overline{\mathbf{H}}_{P E}^{\Theta} \mathbf{a} \\
& \text { s.t. }\left\{\begin{array}{l}
\varphi \mathbf{a}^{T} \mathbf{H}_{U}^{\Theta} \mathbf{a} \geq \rho_{U} \\
|\Theta \mathbf{a}| \leq \mathbb{1} .
\end{array}\right.
\end{aligned}
$$

where $\rho_{U}$ denotes the required SNR of the UE.

Following the same approach as above leads to an analogous result:

$$
\mathbf{H}_{U}^{\Theta} \mathbf{a}=\eta \overline{\mathbf{H}}_{P E}^{\Theta} \mathbf{a}
$$

where $\eta=1 / \lambda$. Similarly, the maximum eigenvalue $\eta_{\max }$ and corresponding eigenvector $\mathbf{a}_{\max }$ can be obtained through the inverse free preconditioned Krylov subspace projection method, and the solution satisfies

$$
\bar{\gamma}_{P E}=\varphi \mathbf{a}^{T} \overline{\mathbf{H}}_{P E}^{\Theta} \mathbf{a}=\varphi \frac{1}{\eta} \mathbf{a}^{T} \mathbf{H}_{U}^{\Theta} \mathbf{a} \geq \frac{1}{\eta_{\max }} \rho_{U} .
$$

From this, we deduce that the minimum $\bar{\gamma}_{E}$ is inversely proportional to the maximum eigenvalue of $\eta_{\max }$. Thus, the optimal vector $\mathbf{a}^{*}$ can be obtained by scaling the corresponding 
eigenvector $\mathbf{a}_{\max }$ such that $\varphi \mathbf{a}^{* T} \mathbf{H}_{U}^{\Theta} \mathbf{a}^{*}=\rho_{U}$. Finally, the optimal beamforming vector $\mathbf{w}^{*}$ can be obtained as $\mathbf{w}^{*}=\Theta \mathbf{a}^{*}$.

If the second constraint in (17b) is not slack, the optimal solution $\mathbf{a}^{*}$ should be solved by a numerical method similar to Algorithm 1, where the left term of the first constraint in (17b) needs to be transformed to an affine function (i.e., convex) by using a first-order Taylor series approximation:

$$
\varphi \mathbf{a}_{k}^{T} \mathbf{H}_{U}^{\Theta} \mathbf{a}_{k}+2 \varphi\left(\mathbf{H}_{U}^{\Theta} \mathbf{a}_{k}\right)^{T}\left(\mathbf{a}-\mathbf{a}_{k}\right) .
$$

We will verify the performances of the proposed ZF beamforming schemes in Section V.

\section{RELAXED ZERO-FORCING BEAMFORMING}

In Section III, we proposed a $\mathrm{ZF}$ beamforming scheme for minimizing the SNRs of the AEDs, while simultaneously maximizing the SNR of the UE and constraining the average SNR of the PEDs. In that context, the ZF beamforming vector should always lie in the null space of the AEDs' channel matrix. However, this condition is sometimes so strict that it makes the $\mathrm{ZF}$ beamformer unable to cope with the PEDs effectively, especially when the number of LED transmitters is not high (i.e., the AED null space is small) or the UE is located at an unfavorable location, e.g., the middle of the LED transmitters. In this case, the area in which the SNR is higher than at the UE can be extensive; therefore, the proposed $\mathrm{ZF}$ beamforming scheme cannot always adequately protect the information transmission against PEDs.

We now propose an alternative approach to cope with this problem. In this method, the optimization goal is to minimize the SOP caused by the PEDs while satisfying positive SNR constraints for the UE and the AEDs, respectively. The main characteristic of the alternative beamforming technique is that the constraints on the SNRs of the AEDs are relaxed, i.e., we let $\gamma_{A E_{p}}>0$ to allow a larger subspace from which to choose feasible beamforming vectors. Thus, we call this strategy relaxed $Z F$ beamforming.

Based on these design features, we formulate an optimization problem minimizing the SOP in (6):

$$
\begin{aligned}
& \arg \min _{\mathbf{w}} 1-\exp \left(-\iint_{\mathcal{A}} \lambda_{P E} \mathrm{~d} \mathbf{x}\right) \\
& \text { s.t. }\left\{\begin{array}{l}
\gamma_{U} \geq \rho_{U} \\
\gamma_{A E_{p}} \leq \rho_{A E}, \quad \forall p \in\left\{1,2, \ldots, N_{A}\right\} \\
|\mathbf{w}| \leq \mathbb{1}
\end{array}\right.
\end{aligned}
$$

where the first and second constraints ensure that the SNRs of the UE and the AEDs satisfy the SNR requirement $\rho_{U}$ and the SNR constraint $\rho_{A E}$, respectively. Without loss of generality, we assume that the same value of $\rho_{A E}$ is set for all the AEDs. To guarantee the PLS of the VLC system, we let $\rho_{U}>\rho_{A E}$, which is consistent with assumption (a) in (6). Then, this optimization problem can be equivalently transformed to

$$
\begin{aligned}
& \arg \min _{\mathbf{w}}|\mathcal{A}| \\
& \text { s.t. }\left\{\begin{array}{l}
\gamma_{U} \geq \rho_{U} \\
\gamma_{A E_{p}} \leq \rho_{A E}, \quad \forall p \in\left\{1,2, \ldots, N_{A}\right\} \\
|\mathbf{w}| \leq \mathbb{1}
\end{array}\right.
\end{aligned}
$$

where $|\mathcal{A}|$ denotes the area of the region $\mathcal{A}$.

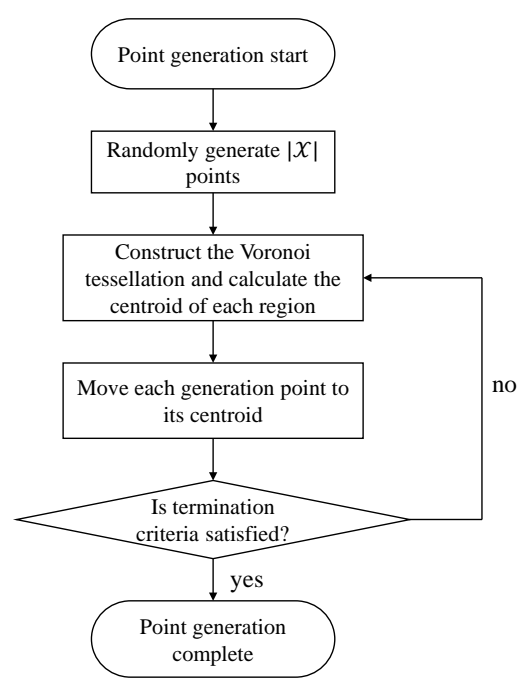

Fig. 3. A flow chart of the point generation procedure using the Lloyd algorithm.

Calculating $|\mathcal{A}|$ analytically is generally intractable. Hence, we exmploy an alternative approach by sampling the SNR space at points $\mathbf{x}_{i} \in \mathcal{X}$, where $\mathcal{X}$ signifies a set of locations in the work plane of the room. An SNR surface according to the receiver locations like Fig. 1 in VLC systems is generally continuous and smooth due to the properties of optical channels, which do not experience fading and largely depend on geometric factors related to indoor VLC environments [23]. Therefore, a small number of discrete points constituting $\mathcal{X}$ would be enough to describe the continuous SNR surface. This approach reduces computational complexity.

\section{A. Sampling the SNR Space}

First, we explain the practical procedure to generate the points $\mathbf{x}_{i} \in \mathcal{X}$ by utilizing Lloyd's algorithm [12]. The number and distribution of $\mathbf{x}_{i}$ affect the efficiency of the optimization; thus, carefully distributing the points is crucial. This is because the area that is likely to experience a high SNR is of primary interest, since the objective is to minimize the SOP by reducing the area $\mathcal{A}$ that retains the high SNR values. If most points are generated in the area in which the SNRs are low, the optimization problem cannot correctly recognize the high SNR region. Thus, we must sample the space more in the high SNR area.

Lloyd's method is an iterative algorithm that can be used to generate a centroidal Voronoi diagram. We can use the algorithm to generate the points $\mathbf{x}_{i} \in \mathcal{X}$ according to the SNR level. This can be achieved by replacing the density function for centroidal Voronoi diagrams with the SNR function $\gamma(\mathbf{x}, \mathbf{w})$ in (7). More specifically, Fig. 3 shows a flow chart of the point generation procedure using the Lloyd method. Observing the figure, we first randomly generate a fixed number of points $\mathbf{x}_{i}$ in the work plane of the room. Then, we construct the Voronoi tessellation based on the sample points and calculate 


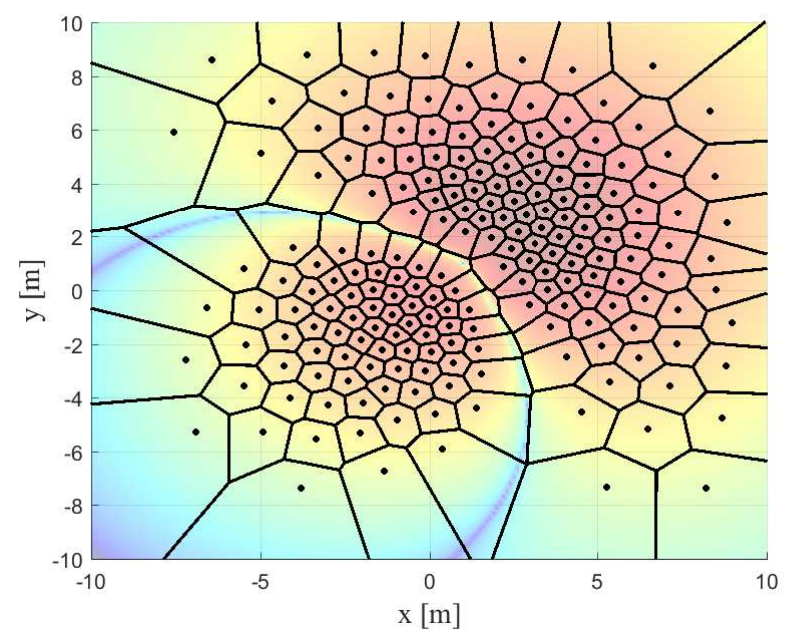

Fig. 4. The points obtained using Lloyd's algorithm with $|X|=200$. $\gamma(\mathbf{x}, \mathbf{w})$ for Fig. 1(a) is adopted.

the centroid of each region in the tessellation using

$$
\mathbf{C}_{i}=\frac{\int_{\mathcal{A}_{\mathbf{x}_{i}}} \mathbf{x} \gamma(\mathbf{x}, \mathbf{w}) \mathrm{d} \mathcal{A}_{\mathbf{x}_{i}}}{\int_{\mathcal{A}_{\mathbf{x}_{i}}} \gamma(\mathbf{x}, \mathbf{w}) \mathrm{d} \mathcal{A}_{\mathbf{x}_{i}}}
$$

where $\mathcal{A}_{\mathbf{x}_{i}}$ denotes the tile region for the generated point $\mathbf{x}_{i}$. Next, we move each point $\mathbf{x}_{i}$ to its centroid $\mathbf{C}_{i}$ and then reconstruct the Voronoi tessellation and so on. The algorithm proceeds iteratively until a termination criterion is satisfied, e.g., the maximum distance moved in any region in an iteration falls below a certain threshold. Fig. 4 shows the generated points (black dots) belonging to $\mathcal{X}$ that result from the proposed algorithm. Note that the points are densely and evenly distributed in the higher SNR area as intended.

\section{B. Optimizing Over the Sampled Space}

Returning to the problem described in (22), by using the points $\mathbf{x}_{i}$ generated by Lloyd's method, we can re-express (22) as

$$
\begin{aligned}
& \arg \min _{\mathbf{w}} \sum_{\mathbf{x}_{i} \in X} \max \left\{\gamma\left(\mathbf{x}_{i}, \mathbf{w}\right)-\gamma_{U}, 0\right\} \\
& \text { s.t. }\left\{\begin{array}{l}
\gamma_{U} \geq \rho_{U} \\
\gamma_{A E_{p}} \leq \rho_{A E}, \quad \forall p \in\left\{1,2, \ldots, N_{A}\right\} \\
\bar{\gamma}_{P E} \leq \bar{\rho}_{P E} \\
|\mathbf{w}| \leq \mathbb{1} .
\end{array}\right.
\end{aligned}
$$

Note that since the objective function in (24a) decreases when $\gamma\left(\mathbf{x}_{i}, \mathbf{w}\right)$ is less than $\gamma_{U}$, the optimization problem aims to find the beamformer that makes the number of points $\mathbf{x}_{\mathbf{i}}$ that retain a higher SNR than $\gamma_{U}$ as few as possible, which naturally reduces $|\mathcal{A}|$ to make it as small as possible. Although it can be seen that the SNRs of PEDs are already taken into account in the objective function, we add the third constraint in (24b). This constraint restricts the average SNR of the PEDs $\bar{\gamma}_{P E}$ to $\bar{\rho}_{P E}$ in order to prevent the area far from the UE, where $\mathbf{x}_{i}$ may not exist, from retaining a very high SNR.
To deal with the function $\max \{\cdot\}$ in the objective, (24) can be equivalently transformed with an auxiliary variable $t_{i}$ for $i \in\{1,2, \ldots,|X|\}$ to yield the problem

$$
\begin{aligned}
& \arg \min _{\mathbf{w}, t_{i} \forall i} \sum_{i} t_{i} \\
& \text { s.t. }\left\{\begin{array}{l}
t_{i} \geq \gamma\left(\mathbf{x}_{i}, \mathbf{w}\right)-\gamma_{U}, \quad \forall i \in\{1,2, \ldots,|X|\} \\
t_{i} \geq 0, \quad \forall i \in\{1,2, \ldots,|X|\} \\
\gamma_{U} \geq \rho_{U} \\
\gamma_{A E_{p}} \leq \rho_{A E}, \quad \forall p \in\left\{1,2, \ldots, N_{A}\right\} \\
\bar{\gamma}_{P E} \leq \bar{\rho}_{P E} \\
|\mathbf{w}| \leq \mathbb{1} .
\end{array}\right.
\end{aligned}
$$

Substituting (3) into (25), the problem can be written as

$$
\begin{aligned}
& \arg \min _{\mathbf{w}, t_{i} \forall i} \sum_{i} t_{i} \\
& \text { s.t. }\left\{\begin{array}{l}
t_{i} \geq \gamma\left(\mathbf{x}_{i}, \mathbf{w}\right)-\varphi \mathbf{w}^{T} \mathbf{H}_{U} \mathbf{w}, \quad \forall i \in\{1,2, \ldots,|X|\} \\
t_{i} \geq 0, \quad \forall i \in\{1,2, \ldots,|X|\} \\
\varphi \mathbf{w}^{T} \mathbf{H}_{U} \mathbf{w} \geq \rho_{U} \\
\varphi \mathbf{w}^{T} \mathbf{H}_{A E_{p}} \mathbf{w} \leq \rho_{A E}, \quad \forall p \in\left\{1,2, \ldots, N_{A}\right\} \\
\varphi \mathbf{w}^{T} \overline{\mathbf{H}}_{P E} \mathbf{w} \leq \bar{\rho}_{P E} \\
|\mathbf{w}| \leq \mathbb{1} .
\end{array}\right.
\end{aligned}
$$

Here, the difficulty of solving (26) arises from the fact that the second term on the right side of the first constraint and the left side of the third constraint yield non-convex sets. Thus, once again, we apply the CCP approach [21]. Given initial feasible points $\mathbf{w}_{0}, t_{i, 0} \forall i$, we transform the nonconvex functions into affine functions by calculating the firstorder Taylor series approximations. We then solve the convex optimization problem. Next, we set the solutions to $\mathbf{w}_{1}, t_{i, 1} \forall i$, and repeat the same procedure until the improvement in the objective value is less than a predefined threshold $\epsilon$. The details of this iterative algorithm are given in Algorithm 2. Since the CCP works in a heuristic manner, the final solution depends on the initial points $\mathbf{w}_{0}, t_{i, 0} \forall i$. Therefore, it is wise to start the algorithm with several initial points and take as the final choice, denoted by $\mathbf{w}^{*}, t_{i}^{*} \forall i$, that which minimizes the objective function.

On the other hand, it is worth mentioning that the CCP has features and advantages that it takes an initial point that significantly affects the final point and that it can retain more information in each of the iterates than other heuristic algorithms since the CCP reserves the information from all the convex components and only linearizes the concave portions [21]. In other words, if the CCP takes an initial point that is feasible and seems close to the optimal point, it is likely to yield a moderately near-optimal performance. In this sense, using the $\mathrm{ZF}$ beamforming vector obtained from (10) to generate the initial point $\mathbf{w}_{0}$ is a practical approach, since the ZF beamformer can initially satisfy the conditions of (26) for reasonable values of $\rho_{U}, \rho_{A E}$, and $\bar{\rho}_{P E}$ and seems close to the optimal point. With this initial point, the optimization procedure (26) gradually changes the ZF beamforming vector into the optimal beamformer $\mathbf{w}^{*}$ of the relaxed ZF beamforming method as the $\mathrm{CCP}$ algorithm iterates, thus minimizing the area that the SNR is higher than 


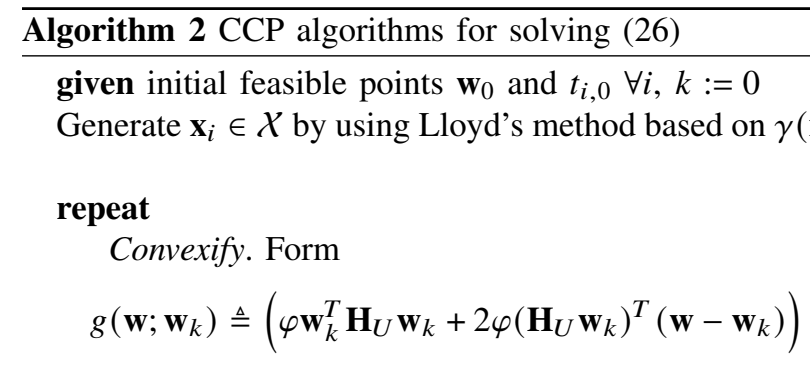

Solve. Set the values of $\mathbf{w}_{k+1}$ and $t_{i, k+1} \forall i$ to solutions of the convex problem

$$
\begin{aligned}
& \min _{\mathbf{w}, t_{i} \forall i} \sum_{i} t_{i} \\
& \text { s.t. }\left\{\begin{array}{l}
t_{i} \geq \gamma\left(\mathbf{x}_{i}, \mathbf{w}\right)-g\left(\mathbf{w} ; \mathbf{w}_{k}\right), \forall i \in\{1,2, \ldots,|X|\} \\
t_{i} \geq 0, \quad \forall i \in\{1,2, \ldots,|X|\} \\
g\left(\mathbf{w} ; \mathbf{w}_{k}\right) \geq \rho_{U} \\
\varphi \mathbf{w}^{T} \mathbf{H}_{A E_{p}} \mathbf{w} \leq \rho_{A E}, \forall p \in\left\{1,2, \ldots, N_{A}\right\} \\
\varphi \mathbf{w}^{T} \overline{\mathbf{H}}_{P E} \mathbf{w} \leq \bar{\rho}_{P E} \\
|\mathbf{w}| \leq \mathbb{1} .
\end{array}\right.
\end{aligned}
$$

Update iteration. $k:=k+1$.

until stopping criterion is satisfied.

\begin{tabular}{|c|c|}
\hline \multicolumn{2}{|l|}{ Room configuration } \\
\hline $\begin{array}{l}\text { Length }(\mathrm{L}) \times \text { Width }(\mathrm{W}) \\
\end{array}$ & $20 \times 20 \mathrm{~m}^{2}$ \\
\hline Height from the work plane $(\mathrm{H})$ & $2.2 \mathrm{~m}$ \\
\hline Number of LEDs per fixture & 9 \\
\hline \multicolumn{2}{|c|}{ LED electrical and optical characteristics } \\
\hline Average optical power per LED & $0.72 \mathrm{~W}$ \\
\hline Optical power / current $\eta$ & 5 \\
\hline Nominal half-intensity angle $\phi_{1 / 2}$ & $60^{\circ}$ \\
\hline Modulation index $\alpha$ & 0.5 \\
\hline \multicolumn{2}{|c|}{ Optical receiver characteristics } \\
\hline Photodetector's responsivity & $0.54 \mathrm{~mA} / \mathrm{mW}$ \\
\hline Lens refractive index $\kappa_{k}$ & 1.5 \\
\hline Noise power $\sigma^{2}$ & $-98.33 \mathrm{dBm}$ \\
\hline Field of View $\Psi_{c}$ & $90^{\circ}$ \\
\hline
\end{tabular}

TABLE II. Simulation parameters.

that of the UE while satisfying all the requirements for the UE, AED, and PED as stated in the constraints.

\section{Numerical Results}

In this section, numerical results are provided to verify the performances of the proposed ZF beamforming and relaxed ZF beamforming schemes. The room configuration and simulation parameters are given in Table II. The $10 \times 10$ transmitter array consists of LEDs that are uniformly distributed on a square lattice in a $20 \times 20 \mathrm{~m}^{2}$ room. The locations of the LED transmitters are given by $\left(x_{T}, y_{T}, 2.2\right)$, where $x_{T}$, $y_{T} \in\{ \pm 1, \pm 3, \pm 5, \pm 7, \pm 9\}$.

Figs. 5(a) and (b) show the SNR values according to receiver locations when the proposed $\mathrm{ZF}$ beamforming and the relaxed $\mathrm{ZF}$ beamforming strategies are adopted. Also, the SNRs corresponding to traditional ZF beamforming [5] are

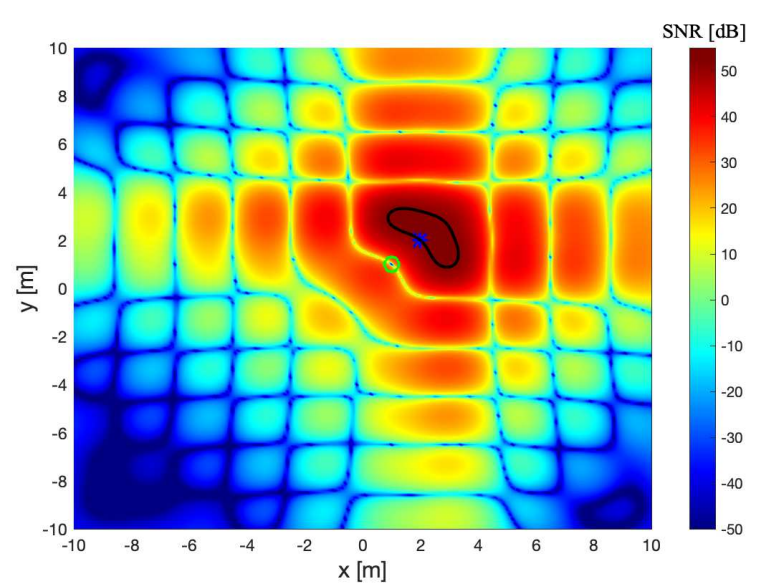

(a) The ZF beamforming of (10).

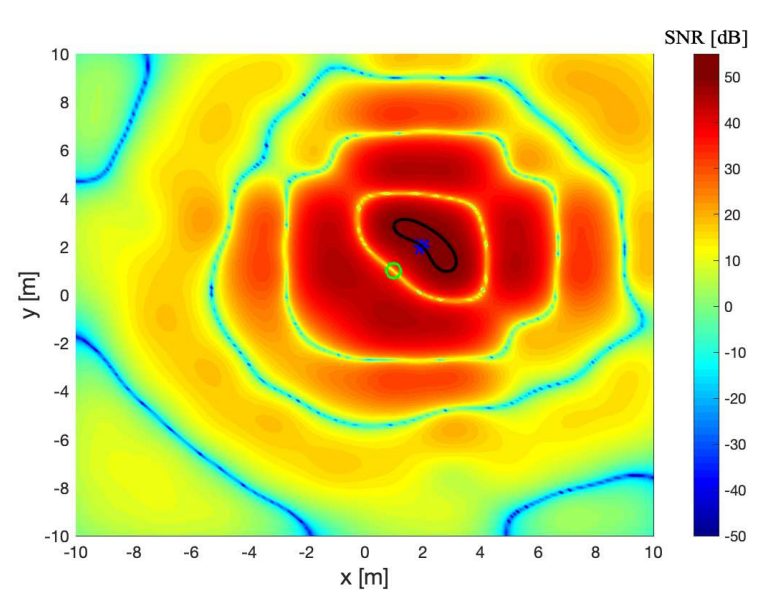

(b) The relaxed ZF beamforming of (21).

Fig. 5. The SNR according to the receiver locations. The $10 \times 10$ LEDs are uniformly distributed on a square lattice. The locations of the UE and the AED are $(2,2)$ and $(1,1)$, respectively. $\rho_{U}=50 \mathrm{~dB}$, $\rho_{A E}=10 \mathrm{~dB}, \bar{\rho}_{P E}=35 \mathrm{~dB}$, and $|\mathcal{X}|=100$ are used.

given in Fig. 1. For all of these results, the UE and the AED are located at $(2,2)$ and $(1,1)$, which are marked by blue and green markers, respectively. Also, the black contours denote the areas in which the SNR is higher than that of the UE. To aid presentation, the figures show the SNR down to $-50 \mathrm{~dB}$, although the SNRs at the AED sites with the traditional ZF beamforming and proposed ZF beamforming schemes are less than this value. The figures show that the contour areas with the proposed ZF beamforming and the relaxed ZF beamforming schemes are $3.05 \mathrm{~m}^{2}$ and $2.04 \mathrm{~m}^{2}$, respectively, which are much smaller than that of the traditional ZF beamforming $\left(7.9 \mathrm{~m}^{2}\right)$. These results verify that the proposed two ZF beamforming schemes can reduce the area in which the PEDs can achieve a higher SNR than the UE while minimizing the SNR of the AED, or at least smaller than the constraint $\rho_{A E}$ in the relaxed case. On the other hand, it is observed that the contour area with the relaxed ZF beamforming in Fig. 5(b) is slightly smaller than that corresponding to the ZF beamforming method shown in Fig. 5(a). As discussed in Section IV, by relaxing the constraint on the SNR of the 


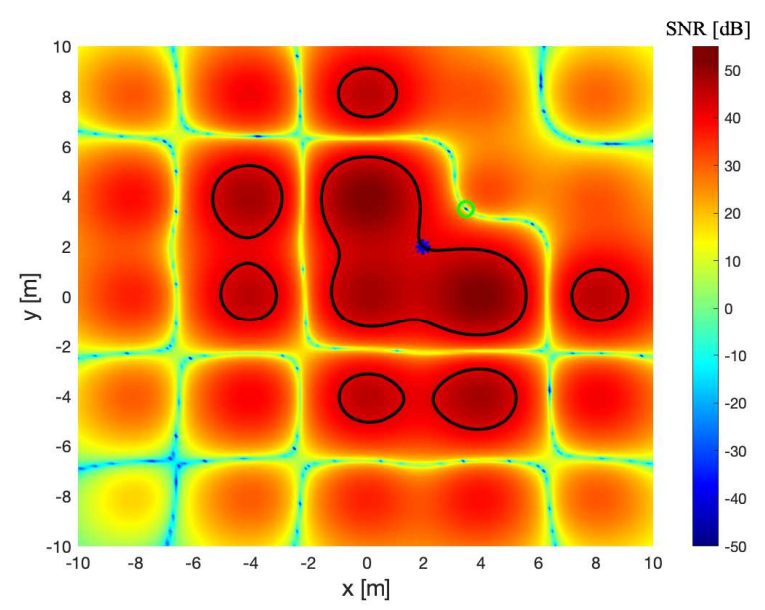

(a) The ZF beamforming of (10).

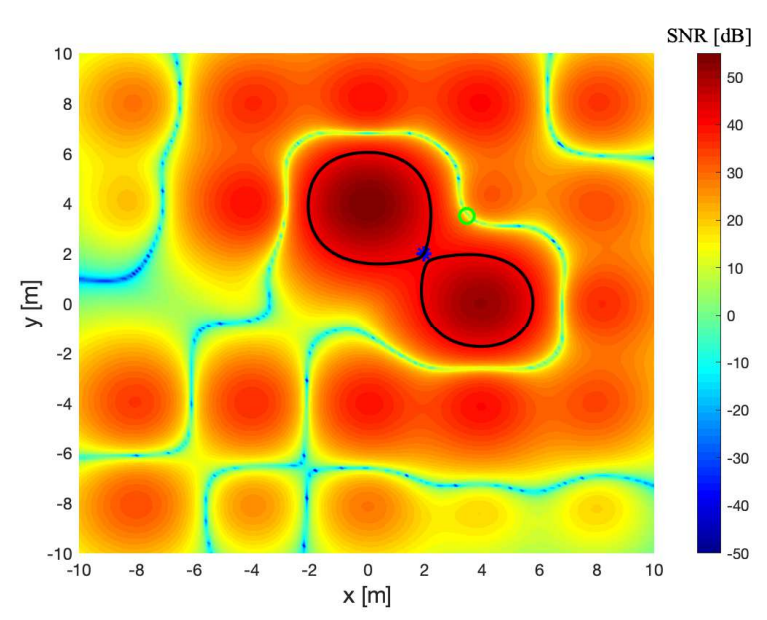

(b) The relaxed ZF beamforming of (21).

Fig. 6. The SNR according to the receiver locations when a smaller number of LEDs, i.e., $N=5 \times 5$, are present. The locations of the UE and the AED are $(2,2)$ and $(3.5,3.5)$, respectively. $\rho_{U}=40 \mathrm{~dB}$, $\rho_{A E}=10 \mathrm{~dB}, \bar{\rho}_{P E}=35 \mathrm{~dB}$, and $|X|=100$ are used.

AED, i.e., from $\rho_{A E}=0$ to $\rho_{A E}=10 \mathrm{~dB}$, the relaxed ZF beamformer can make the contour area smaller than that of the ZF beamforming, accordingly reducing the likelihood of a secrecy outage occuring.

Figs. 6(a) and (b) more clearly show the performance difference between the ZF beamforming and the relaxed beamforming schemes. Here, a smaller number of LEDs, i.e., $N=5 \times 5$, are located at $\left(x_{T}, y_{T}, 2.2\right)$, where $x_{T}, y_{T} \in\{0, \pm 4, \pm 8\}$. Also, the UE is located at $(2,2)$, i.e., the center of four LED transmitters, which is an unfavorable location from the secrecy perspective. Indeed, since the channel gain in (1) largely depends on the distance between the LED and the receiver, the LED transmitters should sufficiently increase the transmit power by adjusting their beamforming weights up to \pm 1 in order to ensure a high SNR at the UE site. However, the increased transmit power would also raise the possibility of being wiretapped by EDs. Also, the condition that the data transmission must be in the null space of the AEDs' channel matrix in the ZF beamforming scheme reduces the size of

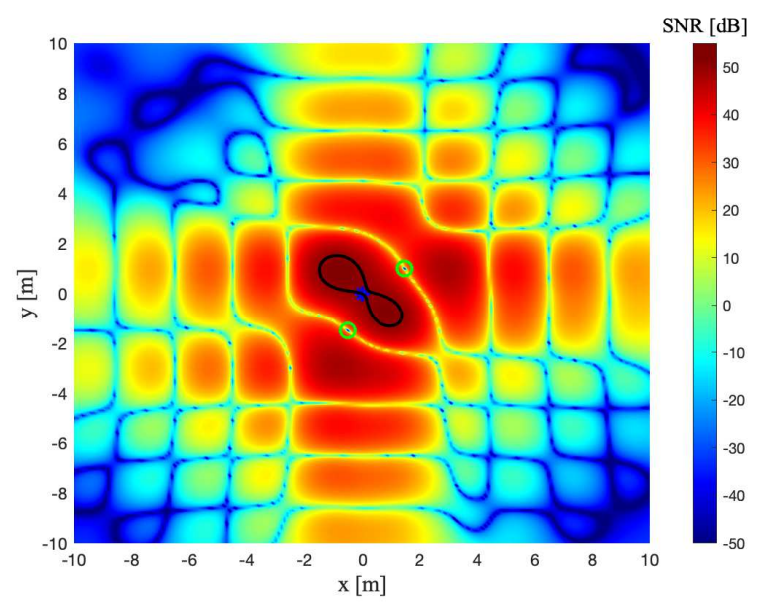

(a) The ZF beamforming of (10).

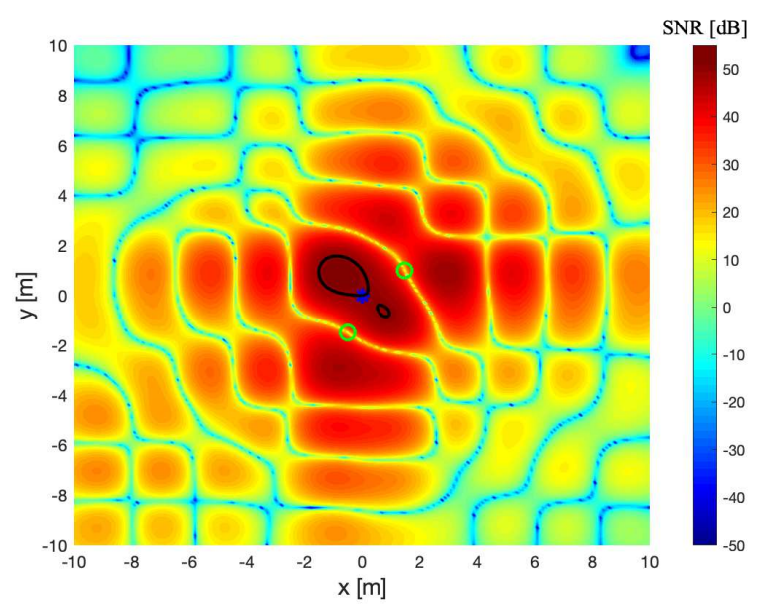

(b) The relaxed ZF beamforming of (21).

Fig. 7. The SNR according to the receiver locations when two AEDs are present. The $10 \times 10$ LEDs are uniformly distributed on a square lattice. The locations of the UE and the two AEDs are $(0,0),(1,1.5)$, and, $(-1.5,-0.5)$ respectively. $\rho_{U}=50 \mathrm{~dB}, \rho_{A E}=10 \mathrm{~dB}, \bar{\rho}_{P E}=$ $35 \mathrm{~dB}$, and $|X|=100$ are used.

the subspace that the beamforming vector can lie within. Therefore, as seen in Fig 6(a), the ZF beamforming scheme yields an extensive contour area $\left(54.4 \mathrm{~m}^{2}\right)$ for this unfavorable secrecy environment, which would result in a high SOP. In contrast, as shown in Fig. 6(b), the relaxed ZF beamforming can somewhat resolve this problem by relaxing the constraint on the SNR of the AED from $\rho_{A E}=0$ to $\rho_{A E}=10 \mathrm{~dB}$ and reduce the contour area to $27.4 \mathrm{~m}^{2}$, which is much smaller than that obtained by ZF beamforming.

Figs. 7(a) and (b) show the SNRs according to the receiver locations when two AEDs are present. Even for multiple AEDs, it is noted that the ZF beamforming scheme in Fig. 7(a) can minimize the SNRs of the two AEDs by aligning the beamforming vectors with the null space of AEDs' channel matrix while maximizing the SNR of the UE. Similarly, the relaxed ZF beamforming scheme restricts the SNRs of the multiple AEDs to lower than $\rho_{A E}=10 \mathrm{~dB}$ while minimizing the contour area in which the PEDs can cause a secrecy outage.

Fig. 8 shows the SOP obtained by Monte Carlo simulation 


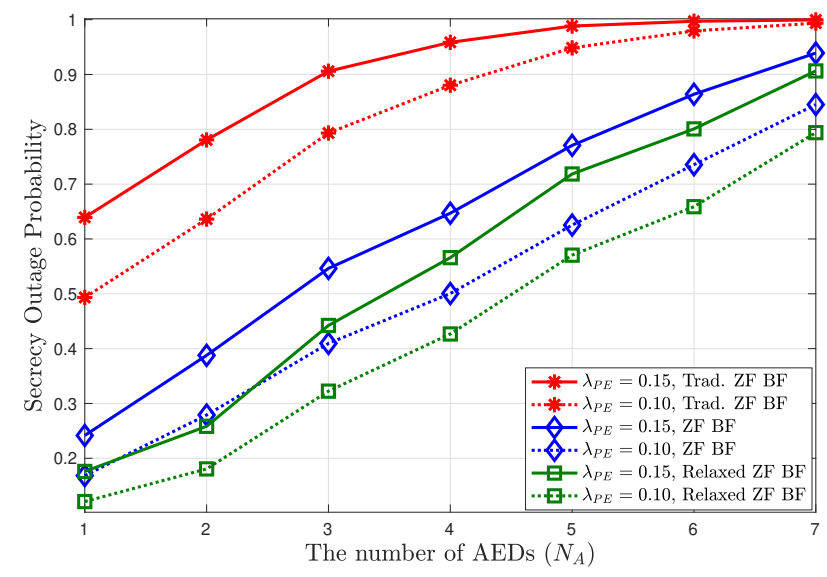

Fig. 8. The SOP plotted as a function of the number of AEDs $N_{A}$. The location of the UE is $(0,0)$, while $N_{A}$ AEDs are randomly located in a circle with radius $3 \mathrm{~m}$ centered at the UE. For ZF beamforming, $\bar{\rho}_{P E}=25 \mathrm{~dB}$ is used. For relaxed ZF beamforming, $\rho_{U}$ is set to be similar to the maximum $\rho_{U}$ obtained by $\mathrm{ZF}$ beamforming for each simulation trial, and $\rho_{A E}=10 \mathrm{~dB}, \bar{\rho}_{P E}=25 \mathrm{~dB}$, and $|X|=100$ are used.

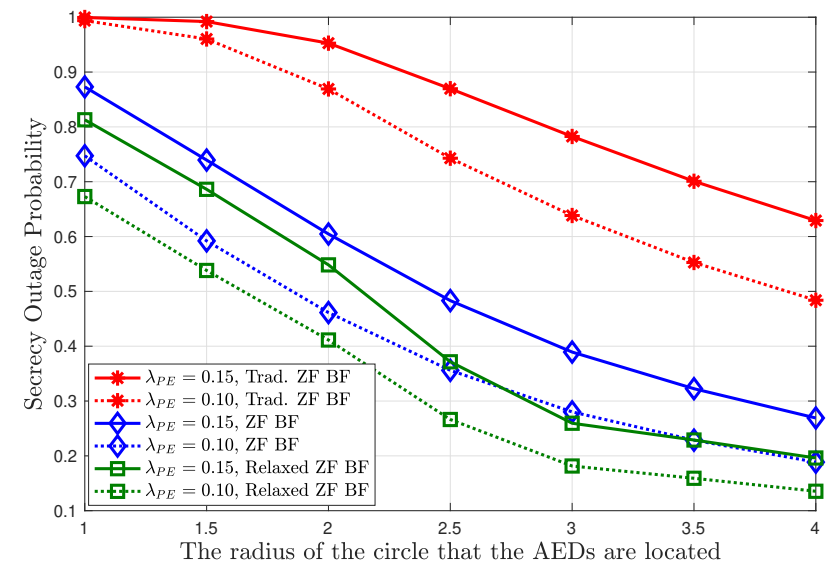

Fig. 9. The SOP plotted as a function of the radius of the circle in which two AEDs are randomly located. The location of the UE is $(0,0)$, and the circle is centered at the UE. For ZF beamforming, $\bar{\rho}_{P E}=25 \mathrm{~dB}$ is used. For relaxed ZF beamforming, $\rho_{U}$ is set to be similar to the maximum $\rho_{U}$ obtained by ZF beamforming for each simulation trial, and $\rho_{A E}=10 \mathrm{~dB}, \bar{\rho}_{P E}=25 \mathrm{~dB}$, and $|X|=100$ are used.

for different densities of PEDs $\lambda_{P E}$ when different numbers of AEDs $N_{A}$ are randomly located around the UE. The UE is located at $(0,0)$, and $N_{A}$ AEDs are randomly located within a circle with radius $3 \mathrm{~m}$ centered at the UE. Also, the SOP corresponding to traditional ZF beamforming [5] is provided as a benchmark. Since [5] considered a single AED, we adopt the ZF beamforming vector $\mathbf{w}=g \Psi_{A E} \Psi_{A E}^{T} \mathbf{h}_{U}$ as in [18] to manage multiple AEDs. The figure shows that the proposed $\mathrm{ZF}$ and relaxed ZF beamforming schemes yield much lower SOPs than traditional ZF beamforming. Also, for all the beamforming schemes, it is noted that the SOP increases as $N_{A}$ increases. For the proposed ZF beamforming, this is because the rank of the null space of the AEDs' channel matrix decreases as more AEDs exist; thus, the subspace that the optimal beamforming vector of (10) maximizing the UE's SNR can lie within also shrinks. Hence, the maximum SNR of the UE achieved by the optimal beamformer is limited, which yields a more extensive contour area. Besides, for the relaxed ZF beamforming approach, a larger number of AEDs impose additional constraints to the optimization problem (21), which contracts the possible vector space for the achievable ZF beamformers minimizing the contour area.

Fig. 9 shows the SOPs obtained by Monte Carlo simulation according to different radii of the circle in which two AEDs are randomly located. The circle is centered at the UE, which is located at $(0,0)$. It is shown that the proposed $\mathrm{ZF}$ and relaxed ZF beamforming methods yield lower SOPs than traditional ZF over all investigated parameters. This result verifies that traditional ZF aims only to lower the AEDs' SNRs without consideration for PEDs; thus, the SOPs with the traditional ZF beamforming approach are shown to be high, especially when the AEDs are likely to be close to the UE. On the other hand, the proposed beamforming schemes can effectively reduce the area in which the SNR is higher than the UE, which results in a lower SOP and suppresses the SNRs of the AEDs.

\section{CONCLUSION}

This paper proposed ZF beamforming schemes for secure VLC systems. Unlike traditional beamforming schemes, which can cope with either AEDs or PEDs, but not both, the proposed ZF beamforming and relaxed ZF beamforming schemes can secure VLC transmissions against AEDs and PEDs simultaneously. With the ZF beamforming scheme, we can design a beamforming vector that lies in the null space of the AEDs' channel matrix, so that the SNRs of AEDs can be completely suppressed in theory while maximizing the SNR of the UE. On the other hand, the relaxed ZF beamforming approach is capable of taking the possible existence of PEDs into account, relaxing the constraint on the SNRs of the AEDs. To reduce the complexity of the relaxed approach, Lloyd's algorithm was used to sample the SNR space, and it was shown through numerical experiments that the new ZF beamforming methods significantly outperform the traditional approach. Given that it both PEDs and AEDs may be present in reality, it can be concluded that the proposed beamforming schemes provide practical approaches for engineering secure VLC systems.

As an extension to this work, it would be interesting to understand how the quantization of beamforming vectors in practice would affect performance. Furthermore, by using similar analytic tools as those used in this paper, an advanced jamming strategy can be developed in which jamming signals are steered along the eigenmode related to the null space of the UE channel and the average channel of the random PEDs to maximize the degree of interference at EDs' sites. In addition, instead of employing a homogeneous PPP for PEDs' locations, it would be an interesting topic to adopt an inhomogeneous PPP model by analyzing the user behavior characteristics and the layout of the room. 


\section{REFERENCES}

[1] D. Karunatilaka, F. Zafar, V. Kalavally, and R. Parthiban, "LED based indoor visible light communications: State of the art," IEEE Commun. Surveys Tuts., vol. 17, no. 3, pp. 1649-1678, 2015.

[2] M. A. Arfaoui, M. D. Soltani, I. Tavakkolnia, A. Ghrayeb, C. Assi, M. Safari, and H. Haas, "Physical layer security for visible light communication systems: A survey," 2019.

[3] M. Bloch and J. Barros, Physical-Layer Security: From Information Theory to Security Engineering. Cambridge University Press, 2011.

[4] A. Mostafa and L. Lampe, "Physical-layer security for MISO visible light communication channels," IEEE J. Sel. Areas Commun., vol. 33, no. 9, pp. 1806-1818, Sept. 2015.

[5] —_, "Physical-layer security for indoor visible light communications," in IEEE ICC in Sydney, Australia, June 2014, pp. 3342-3347.

[6] T. V. Pham and A. T. Pham, "On the secrecy sum-rate of MU-VLC broadcast systems with confidential messages," in 2016 CSNDSP in Prague, Czech Republic, July 2016, pp. 1-6.

[7] T. V. Pham, H. Le-Minh, and A. T. Pham, "Multi-user visible light communication broadcast channels with zero-forcing precoding," IEEE Trans. Commun., vol. 65, no. 6, pp. 2509-2521, June 2017.

[8] S. Cho, G. Chen, and J. P. Coon, "Securing visible light communication systems by beamforming in the presence of randomly distributed eavesdroppers," IEEE Trans. Wireless Commun., vol. 17, no. 5, pp. 2918 2931, May 2018.

[9] _ - "Enhancement of physical layer security with simultaneous beamforming and jamming for visible light communication systems," IEEE Trans. Inf. Forens. Security, vol. 14, no. 10, pp. 2633-2648, Oct. 2019.

[10] A. Arafa, E. Panayirci, and H. V. Poor, "Relay-aided secure broadcasting for visible light communications," IEEE Trans. Commun., vol. 67, no. 6, pp. 4227-4239, 2019.

[11] G. H. Golub and Q. Ye, "An inverse free preconditioned Krylov subspace method for symmetric generalized eigenvalue problems," SIAM J. Sci. Comput., vol. 24, no. 1, pp. 312-334, 2002.

[12] A. Okabe, B. Boots, and K. Sugihara, Spatial Tessellations: Concepts and Applications of Voronoi Diagrams. New York, NY, USA: John Wiley \& Sons, Inc., 1992.

[13] G. Geraci, S. Singh, J. G. Andrews, J. Yuan, and I. B. Collings, "Secrecy rates in broadcast channels with confidential messages and external eavesdroppers," IEEE Trans. Wireless Commun., vol. 13, no. 5, pp. 2931-2943, May 2014.

[14] T. Komine and M. Nakagawa, "Fundamental analysis for visible-light communication system using LED lights," IEEE Trans. Consum. Electron., vol. 50, no. 1, pp. 100-107, Feb. 2004.

[15] Q. Wang, Z. Wang, and L. Dai, "Multiuser MIMO-OFDM for visible light communications," IEEE Photon. J., vol. 7, no. 6, pp. 1-11, Dec. 2015.

[16] S. N. Chiu, D. Stoyan, W. S. Kendall, and J. Mecke, Stochastic geometry and its applications; $3 r d$ ed., ser. Wiley Series in Probability and Statistics. Hoboken, NJ: Wiley, 2013.

[17] A. Baddeley, E. Rubak, and R. Turner, Spatial point patterns: methodology and applications with R. CRC Press, 2015.

[18] A. Khisti and G. W. Wornell, "Secure transmission with multiple antennas I: The MISOME wiretap channel," IEEE Trans. Inf. Theory, vol. 56, no. 7, pp. 3088-3104, July 2010.

[19] S. Boyd and L. Vandenberghe, Convex Optimization. New York, NY, USA: Cambridge University Press, 2004.

[20] J. Money and Q. Ye, "Algorithm 845: EIGIFP: a MATLAB program for solving large symmetric generalized eigenvalue problems," ACM Trans. Math. Softw., vol. 31, pp. 270-279, 012005.

[21] T. Lipp and S. Boyd, "Variations and extension of the convex-concave procedure," Optimization and Engineering, vol. 17, no. 2, pp. 263-287, June 2016.

[22] P. T. Boggs and J. W. Tolle, "Sequential quadratic programming," Acta Numerica, vol. 4, p. 1-51, 1995

[23] J. M. Kahn and J. R. Barry, "Wireless infrared communications," Proceedings of the IEEE, vol. 85, no. 2, pp. 265-298, Feb. 1997. 